both epidemiological and experimental, with regard to the multiple factors involved in the causation of cancer of the alimentary tract. This lecture is reproduced in extenso together with a useful list of references. By contrast the lectures by Renato Dulbeceo (California) on "Viral Carcinogenesis", by George Klein (Stockholm) on "Cancer Immunology" and by Jerome Lejeune (Paris) on "Chromosomal Aberrations and Cancer" are reproduced in much shortened forms, with few references. The final lecture by Severo Ochoa (New York) on "The Molecular Basis of Translation of the Genetic Message" is reproduced in its entirety.

The compromise whereby some of the contributions are reproduced at length, and others not, is on the whole a happy one because the chief value of this book is that it provides a cross section of the state of knowledge as it stood in October 1966 in relation to important aspects of cancer: full length reviews by the same acknowledged experts are available elsewhere.

The official speeches, reproduced verbatim at the end of this volume, serve to emphasize that it is intended to be a historical record. Fortunately, however, these pages of verbal obeisances number only fifteen.

The tenth volume records in abbreviated form the proceedings of nine of the twenty-three panel discussions. Of these nine, the two most important are concerned with the pathogenesis and epidemiology of cancer of the stomach and with the evaluation of controlled clinical trials. The first begins with a detailed study (by Masaru Kuru and Ryozo Sano) of the pathogenesis of gastric cancer in the Japanese: a surprisingly close relation with peptic ulceration is implied by their findings. Later, Takeshi Hirayama reports a negative association between milk consumption and the incidence of gastric cancer in Japan.

The subjects of the remaining seven panel discussions, the proceedings of which are published, include the management of patients with leukaemias, lymphomas and advanced cancers, chemotherapy as an adjuvant to surgery and radiotherapy, anti-smoking campaigns, and nasopharyngeal cancer. Unfortunately the only record of some of the most interesting discussions (for sis") is a note of names of the chairman and principal participants.

As official records of the cancer congress, these two slim, but not inexpensive, volumes serve a purpose. It is understandable that, unless very special preparations are made beforehand, it is neither easy nor necessarily wise to commit to paper all, or even any, of the contributions to a conference or to a discussion. At the same time, a decision not to do so does not imply that, for those present, the discussion was without value.

Theso two reasonably well-edited books provide a useful, though incomplete, record of parts of the Tokyo cancer congress.

F. J. C. RoE cancer detection techniques, cancer hazard from radiation example, panel 10, "Biochemical Aspects of Carcinogene-

electric shocks and following damage to various parts of the nervous system.

Using the technique of duodenal clamping the authors demonstrate that the hypophysial-adrenal system does not contribute to the ulceration. However, their claim that 5-hydroxytryptamine and histamine are not involved cannot be accepted from the statement that "no marked accumulation of either serotonin or histamine in gastric tissue takes place as a result of 'excessive' stimulation of the duodenum". Furthermore, the possibility that enterogastrone, gastrin or other substances might be released is not even mentioned. On the other hand, Professor Anichkov and Dr Zavodskaya present a considerable amount of evidence to show that their methods of inducing gastric ulceration cause stimulation of efferent nerves from the reticular formation in the brain. In rabbits, duodenal trauma alters the electroencephalogram recording, and subdiaphragmatic vagotomy reduces ulcer formation.

Experiments to locate the brain centre involved were made with drugs reported to act at different sites. Depression of lower brain centres with barbiturates reduced ulcer formation, whereas drugs acting on higher centres (chloral hydrate, urethane) gave no protection. The authors cited many experiments to show that the central part of the nervous pathway involved is cholinergic. Antimuscarinic, but not antinicotinic, drugs which penetrate into the brain reduce reflexly induced ulceration. On the other hand, peripherally acting antimuscarinic drugs have no beneficial offect although they markedly reduce gastric secretion of acid and pepsin. The efferent pathway in the reflex is inhibited by drugs which block $\alpha$-adrenergic receptors or prevent transmission in adrenergic nerves.

Professor Anichkov and Dr Zavodskaya used the incorporation of radioactive methionine to show that the development of gastric ulcers is preceded by a reduced synthesis of protein in the mucosa. Reduced protein synthesis also preceded stomach ulceration induced by administration of histamine, reserpine, phenylbutazone and cinchophen. The authors conclude that interference with the synthesis of mucosal protein causes ulcers and that acid and pepsin are only involved after tho lesions have formed. They consider that protein synthesis is affected either directly by nerve impulses or indirectly by a reduced blood supply following vasoconstriction.

The ideas presented in this book are in many cases refreshing. They differ considerably from our usual lines of thought. However, it is sometimes difficult to assess their validity since it is difficult or impossible for most of us to consult the original results in detail. To some extent the book is marred by the paucity of statistical assessment of results, by an incomplete bibliography, by incorrect spelling of the names by which drugs are known in this country, and by the frequent repetition of methods, results and conclusions. Nevertheless, it should be read by everyone interested in the aetiology of gastrointestinal ulceration and in possible methods of treatment.

J. G. Murray

\section{HOW TO GET ULCERS}

\section{The Experimental Basis of Gastric Ulcer Pharmaco- therapy}

By S. V. Anichkov and I. S. Zavodskaya. Translated by Alan Huxley. Pp. vit 171. (Oxford, London and New York: Pergamon Press, 1968.) 75s. net.

THIs is a unique book, translated from Russian, which combines the results of experiments in laboratory animals obtained over many years by the authors and their collaborators. The first experiments described show that gastric ulcers form after irritation of the duodenum by electrical stimulation, by chemicals or by trauma caused by clamping for ten minutes. Gastric ulcers also occur following restraint with or without the added stress of

\section{GENETICS OF STREPTOMYCETES}

\section{Fundamental Genetics of Streptomycetes}

By J. Horvath. Pp. 119. (Budapest: Akademiai Kiado. 1968.) $42 s$.

THIs volume, which might appear from its title to be a text-book summarizing current knowledge of the geneties of members of the genus Streptomyces, turns out to be a kind of thesis describing, for the most part, the author's highly individual approach to an understanding of varia. tion in Streptomycetes. The most remarkable feature of this approach is the deliberate avoidance of precise genetic markers of the kind that have played the dominant role in 